\title{
Patterning vertical sidewall for optical assembly
}

\author{
Taichi Yamamoto, ${ }^{\text {a }}$ Hironori Kubo, ${ }^{\text {a }}$ Shinya Kumagai $\odot,{ }^{b}$ \\ Shigenori Saito $\odot,{ }^{\mathrm{c}}$ Fumitaka Sahara, ${ }^{\mathrm{c}}$ Kenji Wasa, ${ }^{\mathrm{d}}$ and Minoru Sasaki ${ }^{\mathrm{a}, *}$ \\ ${ }^{a}$ Toyota Technological Institute, Department of Advanced Science and Technology, \\ Nagoya, Japan \\ ${ }^{b}$ Meijo University, Department of Electrical and Electronic Engineering, Nagoya, Japan \\ ${ }^{\mathrm{c} A i c e l l o}$ Corporation, Toyohashi, Japan \\ ${ }^{\mathrm{d}}$ Microtex Labs Inc., Tokyo, Japan
}

\begin{abstract}
We report two approaches of the fabrication techniques for three-dimensional patterning across vertical sidewalls based on the photolithography. One approach applies the techniques of spray coating the photoresist and the angled exposure. The supposed application is the microenergy source summing the voltage of silicon $(\mathrm{Si})$ photo cells. Since Si photo cell islands on the buried oxide are isolated from each other, the output voltage is summed well without interacting with the common substrate. Wiring using the vertical sidewalls minimizes the shadow region caused by the metal electrode. Another approach introduces a new sheet, which consists of polyvinyl alcohol (PVA) and polyethylene terephthalate layers. The photoresist film is spin-coated and pasted on the substrate making the bridge over the trench. The pattern of the cantilever overhanging the trench is transferred. Then, PVA is dissolved in water and the resist is developed. When the rinse water dries, the cantilever bends and adheres on the vertical sidewall. The minimum pattern width designed is $10 \mu \mathrm{m}$ and is obtained on the vertical sidewall. (C) The Authors. Published by SPIE under a Creative Commons Attribution 4.0 Unported License. Distribution or reproduction of this work in whole or in part requires full attribution of the original publication, including its DOI. [DOI: 10.1117/1.JOM.1.2.023501]
\end{abstract}

Keywords: optical assembly; three-dimensional photolithography; patterning vertical sidewall; spray coating; sheet with polyvinyl alcohol.

Paper 20012 received Sep. 19, 2020; accepted for publication Jan. 7, 2021; published online Mar. 9, 2021.

\section{Introduction}

Photolithography is advantageous for the efficient production of microdevices. The micropattern is basically transferred from the planer mask to the resist film using UV light. After the resist pattern is obtained, the process reaction of the etching or the deposition occurs at many points at the same time giving a high throughput. The size of the elementary reaction is ultimately small at atomic or molecular levels. Therefore, the finer the mask pattern size, the finer the microstructure that is obtained. If the photoresist is used as the final structural material, there are techniques realizing some unique three-dimensional (3D) structures from the relatively thick planer photoresist. ${ }^{1}$ Ueno et al. ${ }^{2}$ exposed the planer thick negative photoresist SU-8 from the mask with small open holes at inclined angles making the mesh structure. The vertical porous membrane with a height over $50 \mu \mathrm{m}$ is for catching cellular tissues. Keino et al. ${ }^{3}$ reported functional 3D micromesh including titanium dioxide $\left(\mathrm{TiO}_{2}\right)$ microparticles. This 3D micromesh is for microreactors increasing the surface area for the reaction efficiency. This thick resist film is originally planer on the substrate prepared by standard spin coating. In general, the photolithography is valid only for the planer substrate, including the above technique fabricating a 3D resist structure. This is because the uniform resist film and the fine pattern exposure without the diffraction blur are obtained only on the plane. Instead of accepting this planer limitation, integrated circuits (ICs) have realized the higher functionality of the system connecting many elements inside the chip. The layered metal lines on CMOS are fabricated by the planer patterns on the polished surface. ${ }^{4}$ The planer metal lines and the through via insulation layers can match with the request of the IC industry without the active use of the vertical sidewall.

*Address all correspondence to Minoru Sasaki, mnr-sasaki@toyota-ti.ac.jp 
On the other hand, the optical integration requires an additional thing, which is the propagation of the light beam inside the device. Devices matching with the beam size and the positional and the directional alignment along the light path become important. From this view point, the usage of the vertical sidewall higher than the optical beam size becomes attractive. This requires new techniques not only for the resist coating but also for the patterning. Up to now, the wiring using the slant angled sidewall has been well reported. ${ }^{5}$ The vertical sidewalls are the frontier, which is not utilized in the microdevices, ${ }^{6-8}$ since the vertical sidewall cannot be irradiated by the normally incident UV from the exposure equipment. Patterning the 3D samples is one big issue in MEMS research. ${ }^{9}$ Practically, many present devices have vertical sidewalls. The wiring across the vertical sidewall is advantageous for utilizing the device top surface without making the shadow area. There is an approach scanning one focused laser spot using the computer-controlled stage. ${ }^{10}$ The expensive equipment is established but poor in the throughput because the processing proceeds only at a single point and the position control takes time. If the techniques can take advantage of the photolithography of high throughput processing multipoints at the same time, new varieties of devices will be realized having good combinations with the existing planer devices. There are many trials. Toriz-Garcia et al. ${ }^{11}$ introduced a computergenerated hologram mask in conjunction with a diffraction grating for patterning nonplanar substrates. The electrodepositable photoresist was used. Su et al. ${ }^{12}$ applied the self-assembled monolayer coat as a kind of resist and the plasma treatment as a kind of exposure. The contact displacement electroless plating of selective film deposition of $\mathrm{Cu}$ is demonstrated as the 3D wiring. Ji et al. ${ }^{13}$ patterned the sidewalls of a 31-mm-tall and 3.2-mm-wide aluminum (Al) channel using paired silicon ( $\mathrm{Si}$ ) chips. One chip is for through holes as the shadow mask. The other one is for the reflector/diffuser changing the exposure light direction from the channel to the sidewall. Spray coated photoresist is used. An array of 600- $\mu \mathrm{m}$-diameter dimples are demonstrated after the wet-etching of Al. Hirose et al. ${ }^{14}$ reported a self-patterning method. The Si structures prepared by the vertical deep reactive ion etching (RIE) are used as a kind of stencil mask. ${ }^{15}$ The subsequent directional metal deposition over the structure completes the patterning of the deposited material. The trench sidewall profile can provide electrical isolation between the metal line over the concave trench. Westerik et al. ${ }^{16}$ applied the ion beam to etch the upper part of the pillar controlling the inclined angle. The subsequent retraction propagation control of a conformal layer defines the vertical position of the next reaction without using photolithography. Is starts as four layers covering the Si pillars. The material properties of $\mathrm{SiN}$, poly-Si, and $\mathrm{SiO}_{2}$, and isotropic etching and local oxidation are used. Wang et al. ${ }^{17}$ applied the 10 - to $15-\mu \mathrm{m}$-thick flexible polydimethylsiloxane (PDMS) membranes with various holes as the stencil masks for patterning the vertical sidewall combined with the directional metal deposition without using the photoresist.

Here, two applications are considered. (1) One is the microsolar cell harvesting the energy for the sensor node for the Internet of Things microdevices. ${ }^{7}$ For summing the output voltage from the photocells, the electrical connection between cells is indispensable. Figure 1(a) shows the schematic drawing of the photocells, which are square-shaped islands on the buried oxide of the silicon on insulator (SOI) wafer. There is a p-n junction at the top surface of each island. An $\mathrm{Al}$ metal film connects $\mathrm{p}$ - and $\mathrm{n}$-type regions of the neighboring cells. The $\mathrm{Si}$ photo cell islands are

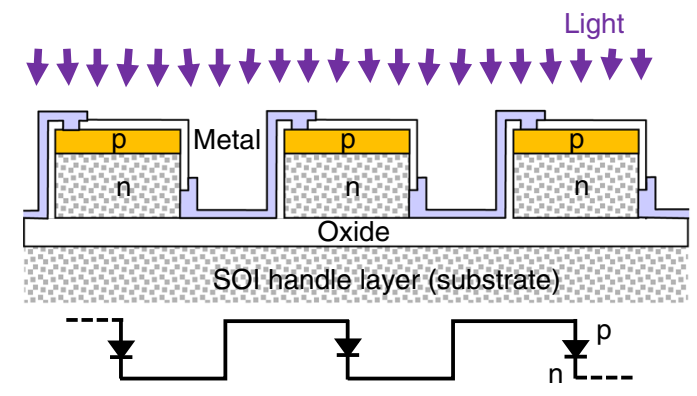

(a)

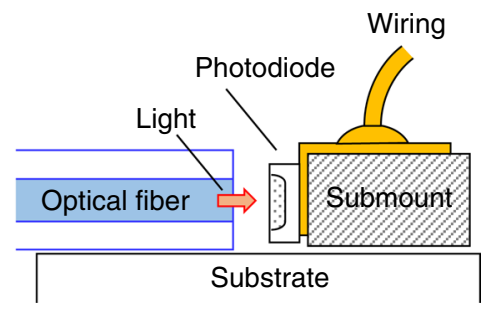

(b)

Fig. 1 (a) Si photo cells with 3D wiring. The sidewall metal does not make the shadow against the incident light. (b) Supposed submount usage in the optical communication device connecting the electrodes between the vertical sidewall and the top flat surface. 
isolated from each other by the oxide layer. Wiring using the vertical sidewalls minimizes the shadow region caused by the metal electrode. (2) Figure 1(b) shows another application, which requires the electrode on the vertical sidewall in the market. ${ }^{18}$ This illustrates the end point of the optical communication network. The optical fiber is set on the planer substrate. The fiber end faces the photodiode. The submount connecting the electrode from the vertical sidewall to the top surface is necessary in the packaging for using a standard wire bonder, since it can only access the top surface. ${ }^{19}$ The industry requires the cost performance. This means a request to the technique applicable without using expensive equipment.

Two different fabrication approaches are described. (1) One approach uses the spray coater and the angled exposure equipment. The absorbent liquid immersion is combined for the exposure. ${ }^{20}$ These apparatuses are special. The material used is standard. (2) Another approach uses a sheet, which has the polyvinyl alcohol (PVA) film on the polyethylene terephthalate (PET) layer. Only this sheet is special. Other materials and the apparatuses are standard.

\section{Processes}

\subsection{Approach Using Spray Coating and Angled Exposure}

The fabrication starts from SOI wafer. After preparing the p-n junction on the device layer of n-type $\mathrm{Si}$ ( 1 to $20 \Omega$-cm) by diffusing boron, the cells are separated from each other using the deep RIE. The cell islands are thermally oxidized covering the surface with 200-nm-thick $\mathrm{SiO}_{2}$. The subsequent process needs $3 \mathrm{D}$ patterning for opening contact holes on the $\mathrm{SiO}_{2}$ cover and for defining sputter-deposited Al metal pattern, since the sample is no longer planer.

Figure 2 shows the schematic drawing of the spray coating. The spray flow is irradiated from the nozzle to the sample. Different from the standard spin coating, the gas flow carries the photoresist as a microparticle. The particles are expected to enter inside the trench. Although the trench is open upside, the actual deposition frequency or the film thickness distribution is influenced by the gas flow and the trench shape. The sprayed resist film becomes thinner at the deeper position inside the trench compared to that at the top surface. This can be explained theoretically considering that the spray flow is blocked against entering inside the trench by the confined air inside the trench. ${ }^{21}$ The subsequent patterning technique is required to allow the resultant nonconformal thickness of the resist.

For irradiating a UV light pattern on the vertical sidewall, the angled exposure is indispensable. Figure 3(a) shows the actual setup. Notice that the angled light generates the reflection, which patterns other surfaces in addition to the first incident surface as shown by the dotted smaller arrows in Fig. 3(b). This reflection generates the problem of patterning the secondary incident surface. Since the sprayed resist film is thinner at the deeper region, the light energy adjusted for patterning the top surface is overdosed for the deeper region. Figure 3(b) includes the thickness information of the sprayed positive resist (Tokyo Ohka Kogyo, TMMR P-W1000).

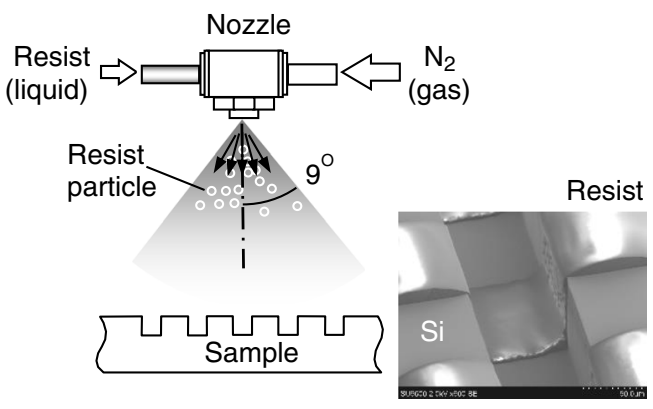

(a)

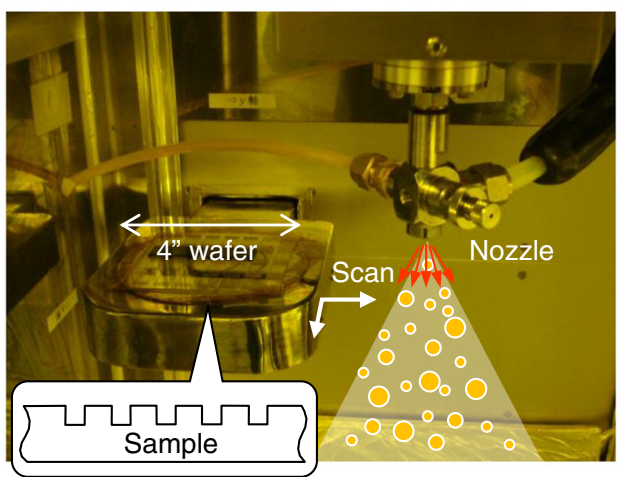

(b)

Fig. 2 (a) Schematic drawing of the spray coating of the photoresist on the trench sample. The photo indicates one example of the resist profile. (b) Photo of the spray coater used. 


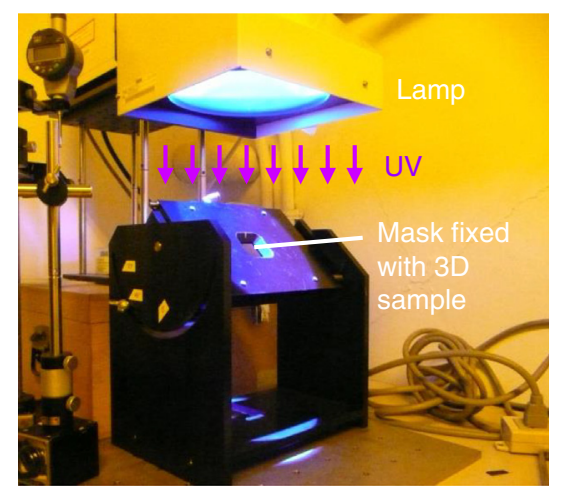

(a)

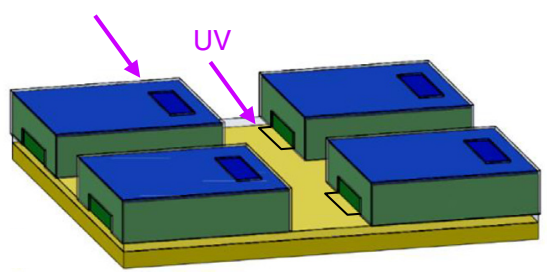

(c)

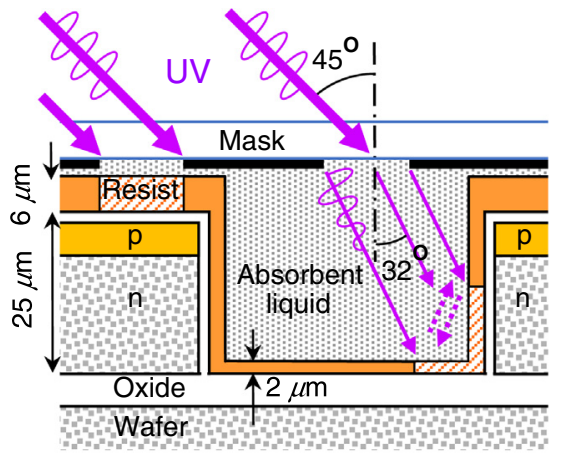

(b)

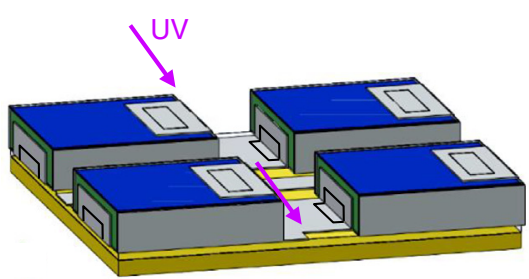

(d)

Fig. 3 Schematic drawing of the angled exposure with the absorbent liquid immersion. The setup is for opening contact holes. Schematic drawing of the angled exposure with the absorbent liquid immersion. The setup is for opening contact holes.

The resist thicknesses at the top and the bottom surfaces are about 6 and $2 \mu \mathrm{m}$, respectively. The appropriate exposure energies are 300 and $150 \mathrm{~mJ} / \mathrm{cm}^{2}$ for the top and bottom surfaces, respectively. The exposure dose is adjusted to $300 \mathrm{~mJ} / \mathrm{cm}^{2}$ for patterning the top surface. This should be decreased to $150 \mathrm{~mJ} / \mathrm{cm}^{2}$ on the bottom of the trench. Therefore, the absorbent liquid immersion is applied. ${ }^{20}$ The trench is filled with the water with black ink for attenuating the incident light power. Since the trench is $50-\mu \mathrm{m}$ wide and $25-\mu \mathrm{m}$ deep, the attenuation ratio can be decided by the concentration of the black ink as the design parameter. The light intensity decreases while UV propagates in the trench. The overdose problem as well as the reflection problem can be removed.

Figure 3(b) includes the information of the optical setup for opening two contact holes (size: $15 \times 50 \mu \mathrm{m}^{2}$ ). One is on the top surface (p-type region). Another is at the sidewall bottom (n-type region). Different from the planer lithography, the resist thickness (6 $\mu \mathrm{m}$ here) has to be taken into account in the mask design since the vertical position shift of the mask causes the lateral position shift of the light pattern. The hole pattern is incident not only on the sidewall, but also on the bottom as shown in Fig. 3(c). This gives the lateral alignment margin of $\pm 4 \mu \mathrm{m}$. The wiring $\mathrm{Al}$ film is sputter-deposited. The thickness on the top surface is about $1 \mu \mathrm{m}$. This thick film is for covering over the corrugations of the scallop on the sidewall. Next is preparing the wiring pattern. Again the angled exposure is applied to pattern the metal lines as shown in Fig. 3(d). Notice that some parts are inevitably in the shadow caused by the neighboring cell, and that the $\mathrm{Al}$ layer remains there. The design allows the remained $\mathrm{Al}$ for realizing the metal line connecting neighboring cells as shown in Fig. 3(d).

\subsection{Approach Pasting a Sheet Including Resist and PVA Layers}

Figure 4 illustrates the principle for patterning the vertical sidewall using the sheet. The resist film is originally pasted over the trench making the bridge. The resist is patterned as the cantilever. The left side in Fig. 4 is the fixed point. The right side is the free end. This shape is formed when the developing process proceeds in the liquid developer. When the rinsing water decreases upon being dried, the surface tension of the water pulls the cantilever film inside the trench. 


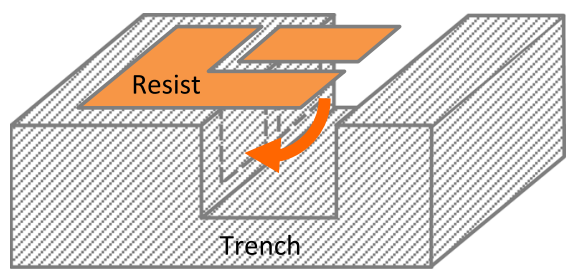

(a)

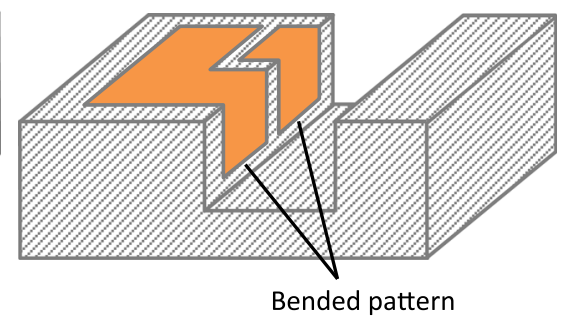

(b)

Fig. 4 Patterning the vertical sidewall by bending a part of the planer pattern. This patterning is for the metal line connecting the top side to the vertical sidewall. Drawings show the conditions (a) before bending (b) after bending.

(1)

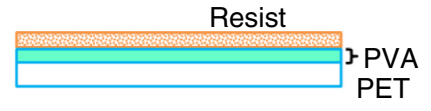

(2)

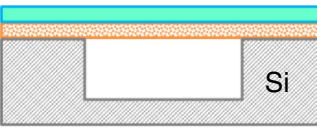

(3)

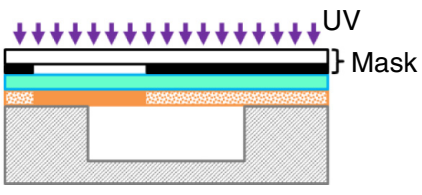

(4)

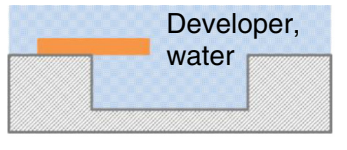

(5)

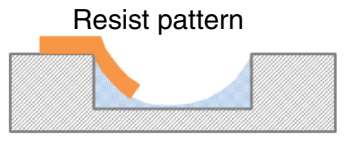

(6)

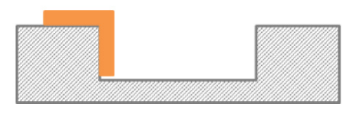

Fig. 5 Fabrication sequence. In this drawing, the negative photoresist is supposed.

Eventually, the film adheres on the vertical sidewall. Once the resist film is on the sidewall, the additional post-baking will strengthen the adhesion of the photoresist to the substrate. Patterning the vertical sidewall is possible using UV irradiation normal to the substrate. The maximum depth of the pattern is the width of the trench corresponding to the aspect ratio of 1 . For realizing the submount with the required height, the trench width becomes the parameter to be designed. Notice that this method is effective for patterning the one side of the sidewall and not for both sides connecting lines as shown in Fig. 3(d).

Figure 5 shows the fabrication sequence starting from the spin coating of the photoresist on the newly introduced sheet (SO sheet, Aicello Corp.) which consists of a PVA layer and a PET substrate. (1) Since the sheet shape is planer during spinning, a uniform thin film is obtained. (2) The resist/PVA layers are pasted on the substrate making the bridge over the trench. The pasting temperature of $90^{\circ} \mathrm{C}$ is somewhat lower than the glass transition temperature of the resist. (3) The patterning defines the cantilever shape to the resist bridge. The mask alignment adjusting to the sample trench is exactly the same operation as that of the standard aligner. (4) PVA is dissolved in water, and the resist film gets the pattern shape being developed. (5) After replacing the developer in the rinsing water, the sample is slowly dried. The surface tension of water attracts the resist cantilever to the trench inside. (6) Finally, the resist film adheres on the vertical sidewall. Notice that all process steps proceed in parallel on the substrate indicating the high throughput.

As shown in Fig. 6, the PVA layer in SO sheet can be peeled from the PET sheet manually. The PVA thickness used is about $35 \mu \mathrm{m}$. Table 1 lists the characteristics of PVA compared with that of the photoresist. PVA is water-solvable and stable against organic solvents (e.g., acetone). ${ }^{22}$ The photoresist is acetone-solvable and stable against water. So, these two materials are opposite in their solvents. The photoresist liquid can be spin-coated on the PVA layer without suffering from the organic solvent. PVA can be selectively removed from the photoresist. This is useful for the process design. ${ }^{23}$ In addition, PVA is transparent. Figure 7 shows the absorption spectrum. The absorption is only $4 \%$ at the wavelength of $400 \mathrm{~nm}$ near to the $\mathrm{i} / \mathrm{g}$ lines used for the patterning. This transparency allows the patterning of the photoresist through the PVA layer. 


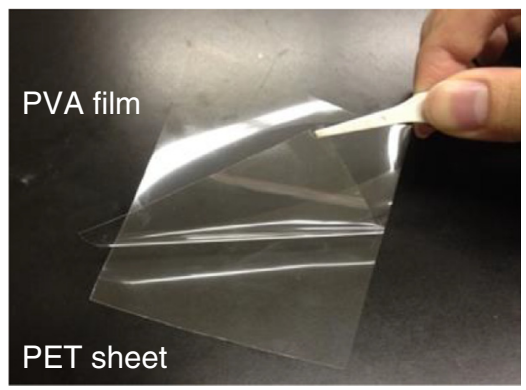

Fig. 6 PVA film on PET sheet. This photo shows the peeled PVA from the PET sheet.

Table 1 Characteristics of PVA and photoresists.

\begin{tabular}{lcc}
\hline \hline Material & PVA & Photoresists \\
\hline Against water & Solvable & Not solvable \\
Against organic solvents & Not solvable & Solvable \\
\hline \hline
\end{tabular}

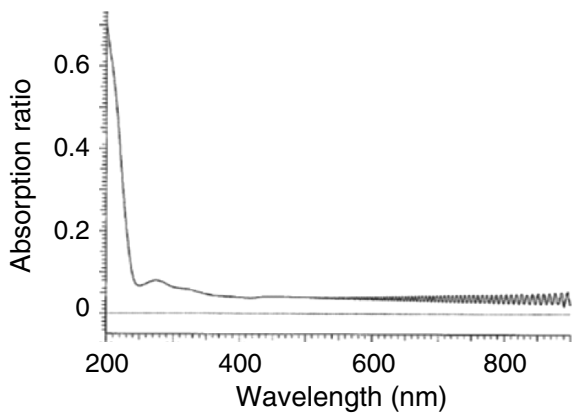

Fig. 7 Absorption spectrum of PVA. PVA is transparent at UV wavelengths. The patterning through PVA film is possible.

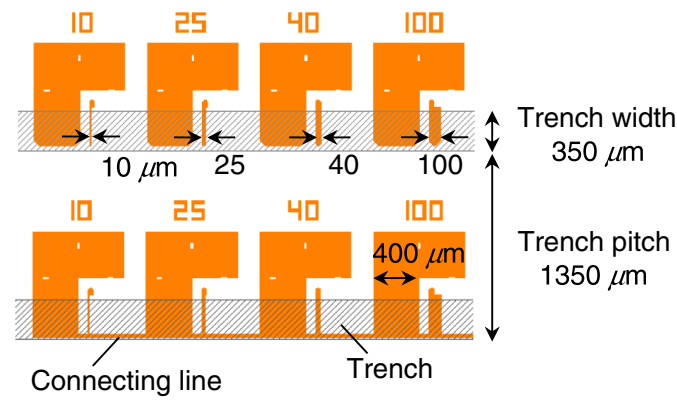

Fig. 8 Photomask pattern relative to the trench. The number on each element means the line width at its right bottom, which will bend to the vertical sidewall.

Figure 8 shows the mask design for patterning the vertical sidewall showing 8 units. A $\Gamma$-shape pattern has $400-\mu \mathrm{m}$ width for all units. The right-side I-shape thinner pattern has widths of $10,25,40$, and $100 \mu \mathrm{m}$. The maximum width examined is $350 \mu \mathrm{m}$. The cantilever length is $315 \mu \mathrm{m}$ (the length from the trench edge). The trench width is $350 \mu \mathrm{m}$. Sixteen parallel trenches are prepared by the half-depth dicing of the substrate (size: $1 \times 2 \mathrm{in}^{2}{ }^{2}$ ). Thus, the sidewall surface is relatively rough. The trench width and depth are both $350 \mu \mathrm{m}$ in design. One corner is chamfered using the bevel blade. The substrate material is alumina or Si. 


\begin{tabular}{|l||c|c|}
\hline Photoresist & Negative & Positive \\
\hline Product used & TMMR SA390N & AZ1500 \\
\hline Base polymer & $\begin{array}{c}\text { Poly(hydroxyl } \\
\text { styrene) }\end{array}$ & $\begin{array}{c}\text { Typical g/i-line } \\
\text { photoresist } \\
\text { novolak }\end{array}$ \\
\hline $\begin{array}{l}\text { Young's modulus (MPa) at } \\
\text { room temperature }\end{array}$ & $\begin{array}{c}250 \\
\text { After cross-linking } \\
\text { reaction }\end{array}$ & 5000 \\
\hline Adhesion & Better & $\mathrm{Bad}$ \\
\hline Temperature at pasting step & $90{ }^{\circ} \mathrm{C}$ & $90{ }^{\circ} \mathrm{C}$ \\
\hline
\end{tabular}

Fig. 9 Characteristics of the photoresists used.

The positive photoresist used is AZ-1500 (AZ Electronic Materials), $38 \mathrm{cp}$. The spin-coating is at $3000 \mathrm{rpm}$ giving a thickness of about $2.4 \mu \mathrm{m}$. The exposure dose is $100 \mathrm{~mJ} / \mathrm{cm}^{2}$. The negative photoresist used is TMMR SA390N (Tokyo Ohka Kogyo Co., Ltd.), $100 \mathrm{cp}$. This resist is softer and adhesive to the sample. As shown in Fig. 9, Young's modulus is $250 \mathrm{MPa}$, which is only $1 / 20$ compared to that of the positive resist. The value before the cross-linking reaction will be smaller than that. The spin-coating is at $2000 \mathrm{rpm}$. The resist thickness is about $5 \mu \mathrm{m}$. The exposure dose is $200 \mathrm{~mJ} / \mathrm{cm}^{2}$. The negative photoresist without the exposure dissolves in the developer. This is good for removing the residue and useful for the thick film.

\section{Results}

\subsection{Pattern Obtained by Spray Coating}

Figures 10(a) and 10(b) show the whole device of the photo cells connected in series. The $5 \times 5$ photo cell array is inside the area of $1.5 \times 1.5 \mathrm{~mm}^{2}$. The single cell size is $250 \times 250 \mu \mathrm{m}^{2}$ with the height of $25 \mu \mathrm{m}$. The Al pattern on the top surface is $35 \times 70 \mu \mathrm{m}^{2}$, which is $3.9 \%$ of the top area of the photo cell. The aspect ratio of the separation trench is 0.5 . The outside larger islands with numbers are electrodes for probing. The voltage and the current can be measured for each row. The pattern on the vertical sidewall is $70-\mu \mathrm{m}$ wide for all cells. The connection between columns is the rounding path on the oxide bottom layer. This design is for simplifying the angled exposure. This rounding path on the bottom is $20-\mu \mathrm{m}$ wide at the center of the $50-\mu \mathrm{m}-$ wide trench. The smaller 5 square $\left(100 \times 100 \mu \mathrm{m}^{2}\right)$ cells for each column are the bypass diodes. Figure 10(c) shows the magnified view. The angled exposure is single UV irradiation from one side. The resist pattern for $\mathrm{Al}$ etching is on one sidewall, and the $\mathrm{Al}$ film remains on the other sidewall as indicated in Fig. 10(d). Figures 10(e) and 10(f) show the sidewall patterns viewed from the opposite side from others [Figs. 10(b)-10(d)]. Figure 10(f) shows the close-up view of the contact electrodes at the sidewall and the top surface. The contact holes are covered by $\mathrm{Al}$ film. The electrical connection from end to end is successfully obtained with a yield of about $50 \%$ at present.

Figure 11 shows the $I-V$ curve for 10 cells in $5 \times 5$ arrays. The light illumination is from the white light-emitting diode (measured to be about $4 \mathrm{~mW} / \mathrm{mm}^{2}$ supposing a 550 -nm wavelength). Under the light illumination, the average open voltage is $0.241 \mathrm{~V} /$ cell, which indicates that there is a margin for improving the quality of the $\mathrm{p}-\mathrm{n}$ junction.

\subsection{Pattern Obtained by Sheet}

\subsubsection{Positive resist}

Figure 12(a) shows SEM images of the patterning result obtained by the sheet using the positive resist. The vertical pattern is obtained on the sidewall. The pattern on the top surface is stable and working as the fixed point for the cantilever. The fixed point [indicated by the white arrow in 


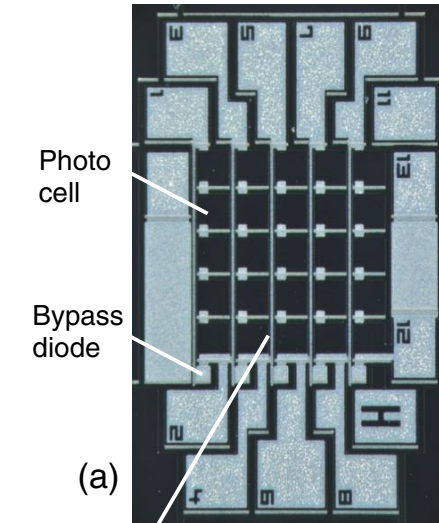

Rounding path $1 \mathrm{~mm}$
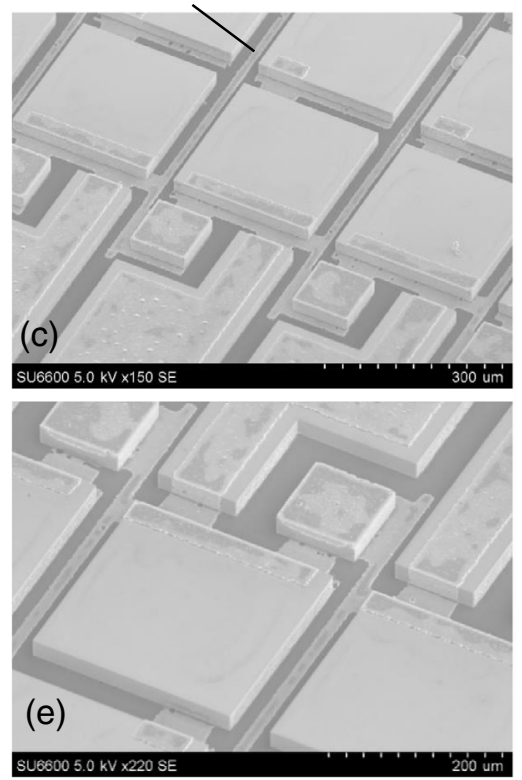
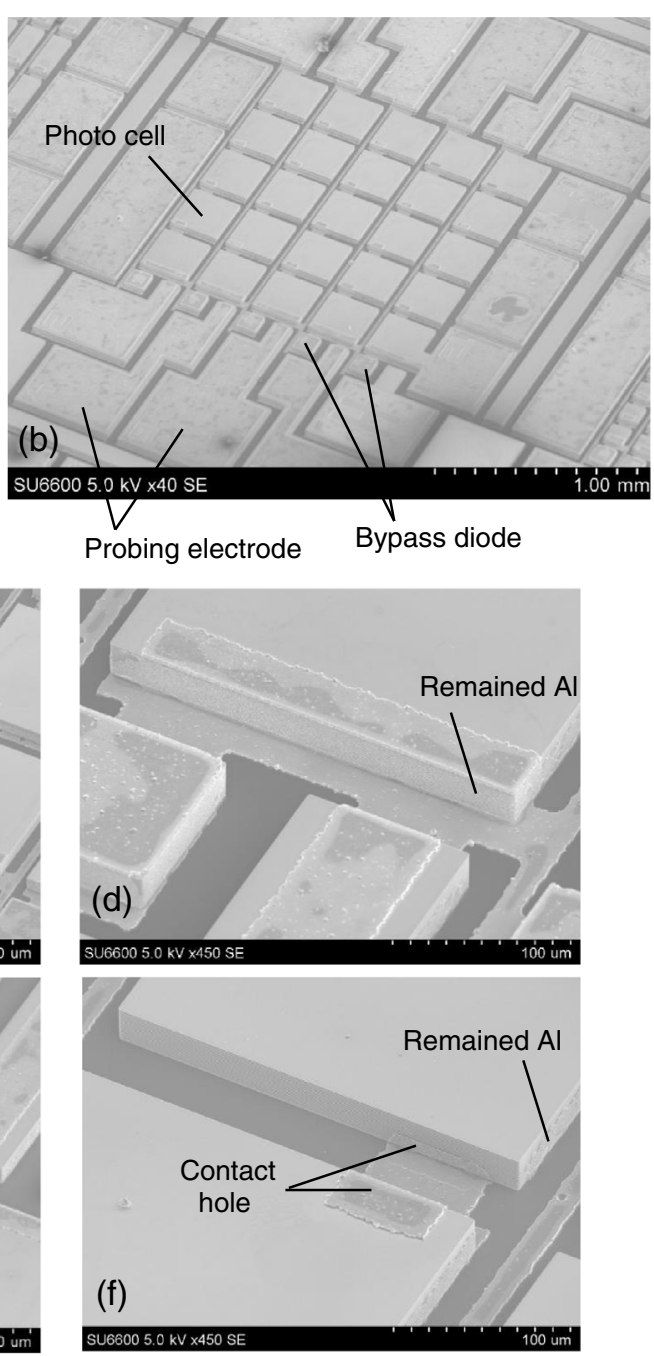

Fig. 10 SEM images of the photo cells with 3D wiring across vertical side walls. (a), (b) Whole image of $5 \times 5$ cells. $1 \times 5$ cells are one group. (c), (d) Magnified view of photo cells and the bypass diode. (e), (f) Close-up views from the opposite angle observing contact holes.

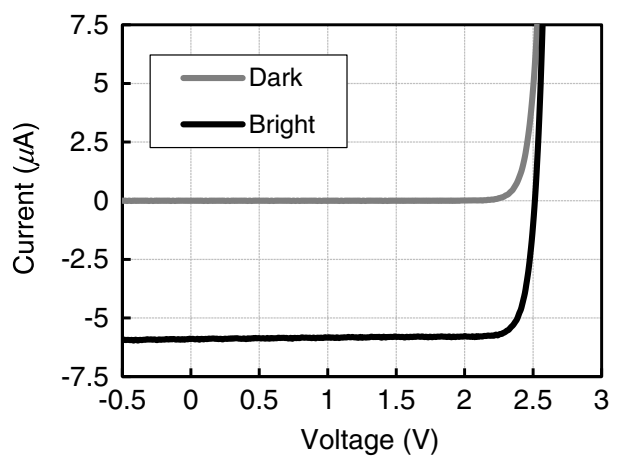

Fig. 11 Typical $I-V$ curves of 10 cells connected in series from $5 \times 5$ array. The short-circuit current $I_{\mathrm{sc}}$ is $-5.89 \mu \mathrm{A}$. The open-circuit voltage $V_{\mathrm{oc}}$ is $2.51 \mathrm{~V}$. The fill-factor defined by $V_{\mathrm{mp}} I_{\mathrm{mp}} / V_{\mathrm{oc}}$ $I_{\mathrm{sc}}$ is $0.827 . I_{\mathrm{mp}}$ and $V_{\mathrm{mp}}$ are the current and the voltage operating point, respectively, which give the maximum power output, where $V_{\mathrm{mp}}$ is $2.37 \mathrm{~V}$ and $I_{\mathrm{mp}}$ is $-5.16 \mu \mathrm{A}$. 

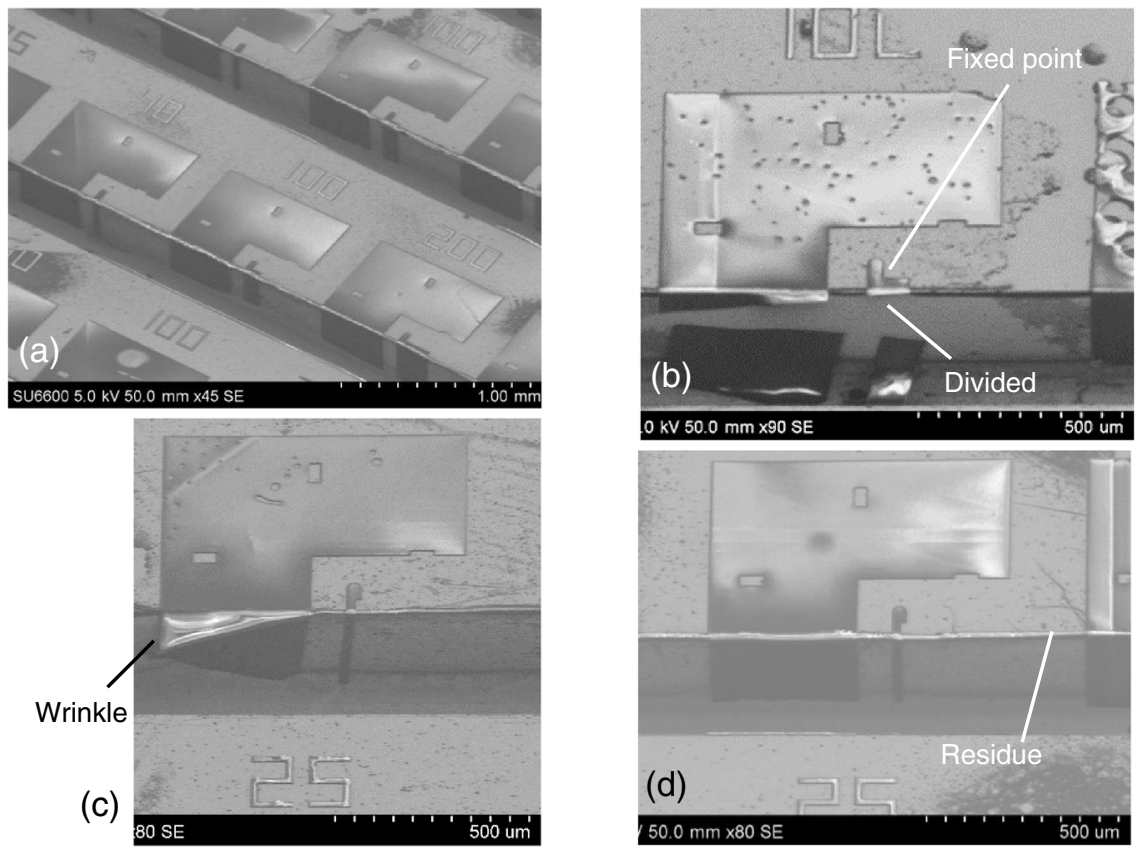

Fig. 12 (a) Patterned positive resist connecting from the top surface to the vertical sidewall. (b) Resist film divided from the corner edge. (c) Resist film pasted on the sidewall with the wrinkle. (d) Corner edge with the local resist residue.

Fig. 12(b)] is stable even if its area $\left(40 \times 60 \mu \mathrm{m}^{2}\right)$ is smaller compared to that of the cantilever. Figure 12(b) shows the film divided from the corner edge. This is the main failure mode. This can be explained by the relatively thin edge and the material fragility. Figure 12(c) shows the resist film fixed with the wrinkle. This indicates the importance of the quiet water condition. Figure 12(d) shows the remaining resist at the corner edge of the trench. This will be caused by the locally thickened resist and the underdosed UV exposure for patterning the positive photoresist. Figure 13 shows the illustration for explaining the above phenomena. The resist material can migrate at the corner from the surrounding area during the heating step for pasting the film (step 2 in Fig. 8).

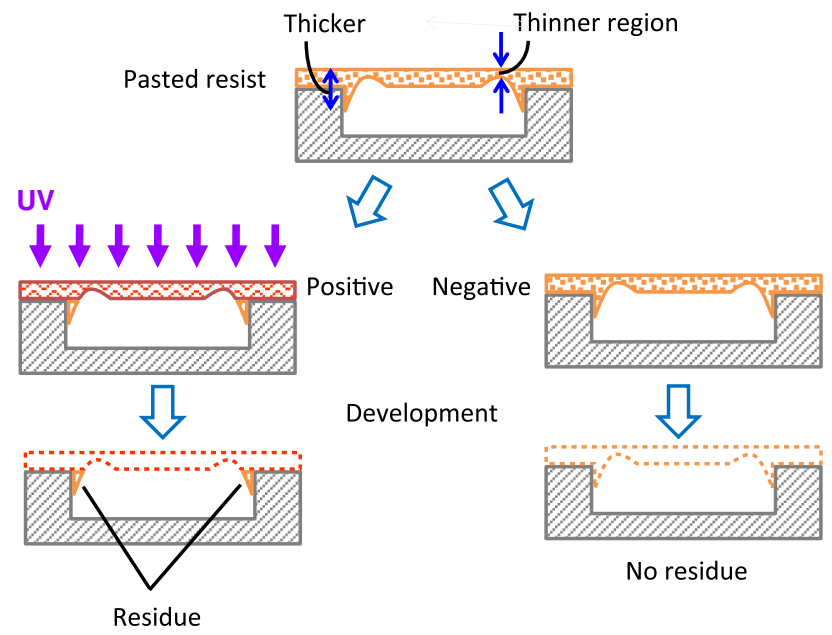

Fig. 13 Comparison of the positive and negative photoresists inside the bridge for avoiding the residue. 


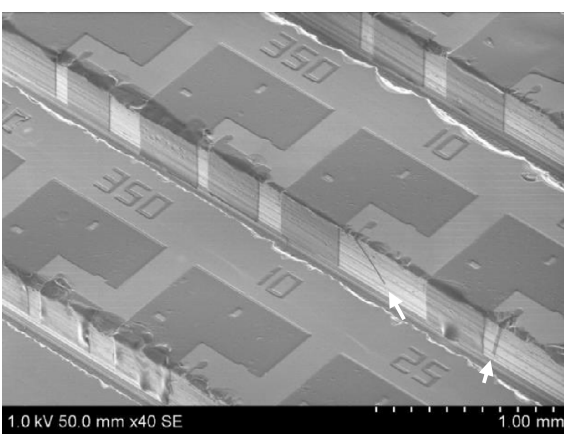

(a)

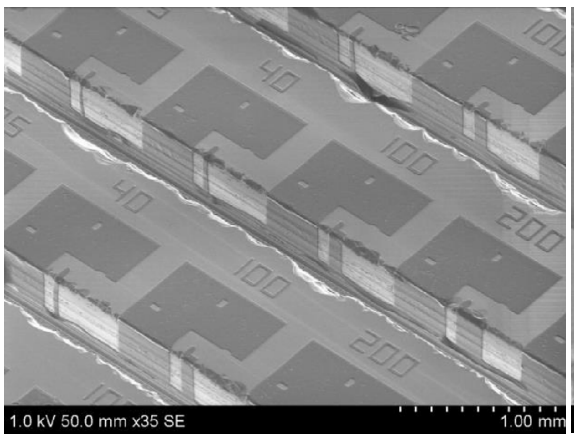

(c)

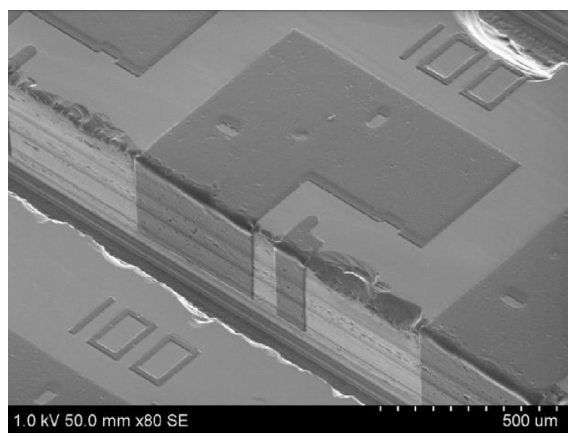

(b)

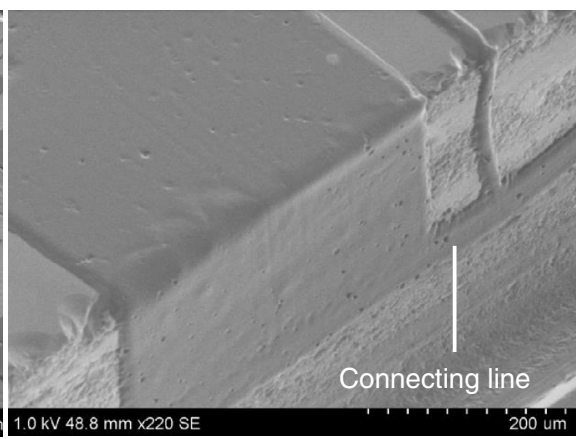

(d)

Fig. 14 Patterned negative resist connecting from the top surface to the vertical sidewall. (a), (b) Pattern having the design of the individual cantilever. (c), (d) Pattern having the design with connecting line between cantilever ends. This line width is $45 \mu \mathrm{m}$. The photos are obtained at different positions from the same substrate.

\subsubsection{Negative resist}

Figure 14(a) shows the pattern prepared using the negative resist. The pattern from the top surface to the sidewall is well obtained. After the rinsing water is removed from the dish, the water remains in the trench. The substrate is continuously dried at room temperature for about $5 \mathrm{~min}$. The rinse water drying speed is necessarily slow, especially for the narrower pattern. The wider pattern (e.g., 400- $\mu \mathrm{m}$ width) is stable in the direction to the bottom. Since each cantilever separates individually, the narrower pattern (e.g., 10- or 25- $\mu \mathrm{m}$ width) tends to be slanted as shown in the white arrows in Fig. 14(a). The condition of the water in the trench may influence the result. The undermost trench in Fig. 14(a) has the vertical patterns with wrinkles. This may be the influence of the local water flow in this trench. Figure 14(b) is the magnified image of 100- $\mu \mathrm{m}$ width pattern at other position in the substrate. Figure 14(c) shows the pattern having the connecting line between the free ends of the cantilevers. The pattern direction is stabilized to be straight vertical even for the $10-\mu \mathrm{m}$-wide line. There is the design variation of the connecting line width to be 15,30 , and $45 \mu \mathrm{m}$. For all line widths, the stabilization effect seems to be same. Figure 14(d) shows the magnified corner with a $10-\mu \mathrm{m}$-wide line. There is no residue at the corner. This is in contrast to Fig. 12(d), which is the case using the positive resist. The resist thickness seems to be same at the top surface, the corner, and the sidewall. This is also in contrast to the inset of Fig. 2(a) obtained using the spray coating.

\subsubsection{Stencil mask using positive resist}

Based on the fact that the positive resist is relatively rigid for folding, this material is rather useful for the stencil mask. The process revision is changing the mask tone making the holes in the resist bridge and increasing the resist thickness to about $7.1 \mu \mathrm{m}$. Figure 15 shows the obtained 


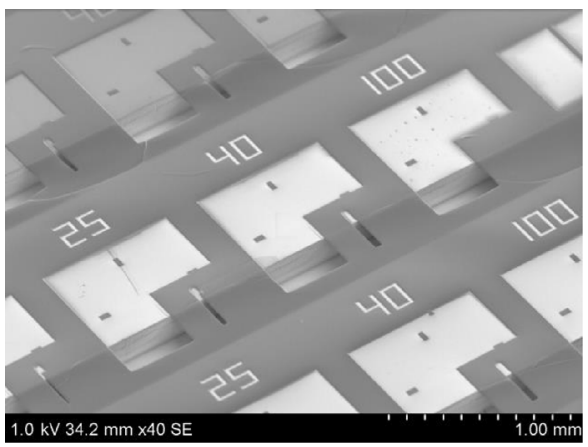

(a)

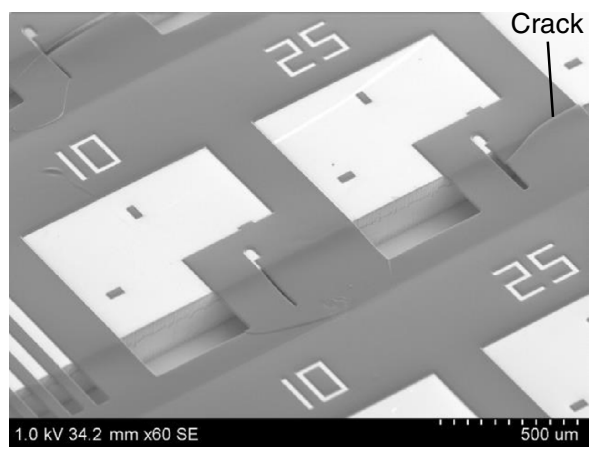

(b)

Fig. 15 Patterned positive resist as the stencil mask over the trench. (a) Reversed tone compared to Fig. 14. (b) Another part around the corner edge of the trench with the cracks in the film.

pattern. The clear hole pattern is well obtained. Since this resist film contacts on the sample surface, this is ideal for minimizing the blur of the metal deposition at the subsequent process step. The membrane over the trench somewhat deflects. This can be explained by the deformation due to the surface tension of the rinsing wafer. Inside the resist membrane, some cracks are observed as shown in Fig. 15(b). They will be removed with the thicker resist membrane or the gentle drying condition reducing the surface tension. There is no residue of the photoresist as seen in Fig. 12(d). The metal deposition from the angled direction using such resist mask will give the $3 \mathrm{D}$ wiring across the vertical sidewall.

\section{Conclusions}

The photolithography-based two approaches for patterning the vertical sidewall are described. (1) Spray coating enables 3D wiring left to right across vertical sidewalls for summing the output voltage of Si photo cells on the isolation oxide layer. The trench for separating the photocells has the aspect ratio of 0.5 . This value is reasonable for balancing the coating and exposure difficulty. The patterned line width is $70 \mu \mathrm{m}$. Wiring using the vertical sidewalls can minimize the shadow. This is important for the photocell. A 3D wiring technique allows the use of photocell materials of $\mathrm{Si}$ and $\mathrm{SiO}_{2}$, which can realize good isolation, but have been difficult to use for the series connection. (2) The sheet, which consists of PVA and photoresist layers, can open the way for patterning the vertical sidewall without using special equipment. In the process, PVA seems to work as the cushion, and the spin-coated thin photoresist is protected. This is advantageous for the fine patterning. When the resist film is pasted on the substrate with the trench, it becomes the bridge. Using the aligner, the mask pattern is transferred to the PVA/resist layers. The patterned cantilever overhangs the trench. After the development and drying the rinsing water, the cantilever bends around the trench corner and adheres on the vertical sidewall. For this bending, the negative photoresist having the lower Young's modulus is better than the positive one. The positive resist is rather promising as the stencil mask directly fixed on the sample surface. The minimum pattern width designed is $10 \mu \mathrm{m}$ and is confirmed.

\section{Acknowledgments}

Part of this research was supported by MEXT Nanotechnology Platform Japan (Grants No. F-17TT-0002 and No. F-17-TT-0023) and Aichi Science and Technology Foundation, Japan.

\section{References}

1. A. del Campo and E. Arzt, "Fabrication approaches for generating complex micro- and nanopatterns on polymeric surfaces," Chem. Rev. 108, 911-945 (2008). 
2. H. Ueno, K. Yamada, and T. Suzuki, "Integration method of microchannel and vertical micromesh structure for three-dimensional cell culture using inclined exposure and inclined oxygen ashing," Micromachines 9, 681 (2018).

3. S. Keino et al., "Fabrication of catalyst-functionalized three-dimensional micromesh structures," Jpn. J. Appl. Phys. 47(6), 5204-5207 (2008).

4. P. F. Van Kessel et al., "A MEMS-based projection display," Proc. IEEE 86(8), 1687-1704 (1998).

5. N. P. Pham et al., "A micromaching post-process module with pattern transfer in deep cavities for RF silicon technology," in Tech. Digest. 14th IEEE Int. Conf. Micro Electro Mech. Syst., pp. 345-348 (2001).

6. H. Morii et al., "Proposal of high-density packaging construction and conductive pattern forming method on vertical wall using spray coating technology," IEEJ Trans. Sens. Micormach. 131, 40-44 (2011).

7. S. Kumagai et al., "3-D wiring across vertical sidewalls of Si photo cells for series connection and high voltage generation," in Tech. Digest. 25th IEEE Int. Conf. Micro Electro Mech. Syst., pp. 60-63 (2012).

8. T. Yamaguchi et al., "Thermocouples fabricated on trench sidewall in microfluidic channel bonded with film cover," Jpn. J. Appl. Phys. 54, 030219 (2015).

9. I. El-Chami et al., "A silicon vector light sensor for proximity sensing applications," in Proc. 19th Int. Conf. Solid-State Sens., Actuators and Microsyst., T1C.004, pp. 163-166 (2017).

10. MIPTEC, "Panasonic industrial devices," http://www3.panasonic.biz/ac/j/tech/mid/miptec index.jsp.

11. J. J. Toriz-Garcia et al., "Vertical tracks on the sidewall of a silicon die using 3D holographic photolithography," J. Micromech. Microeng. 21, 085034 (2011).

12. W.-S. Su, M.-S. Tsai, and W. Fang, "A three-dimensional microfabrication technology on highly structured surfaces," Electrochem. Solid-State Lett. 10(1), H16-H19 (2007).

13. C.-H. Ji, F. Herrault, and M. G. Allen, "Sidewall lithography of micron-sized features in high-aspect-ratio meso-scale channels using a three-dimensional assembled mask," Micro Nano Syst. Lett. 2(1), 6 (2014).

14. K. Hirose, F. Shiraishi, and Y. Mita, "A simultaneous vertical and horizontal self-patterning method for deep three-dimensional microstructures," J. Micromech. Microeng. 17, S68-S76 (2007).

15. A. Tixier et al., "A silicon shadow mask for deposition on isolated areas," J. Micromech. Microeng. 10, 157-162 (2000).

16. P. J. Westerik et al., "Sidewall patterning — a new wafer-scale method for accurate patterning of vertical silicon structures," J. Micromech. Microeng. 28, 015008 (2018).

17. H. Wang, A. Chakraborty, and C. Luo, "Fabrication of Au micropatterns on vertical Si sidewalls using flexible PDMS shadow masks," J. Micromech. Microeng. 20, 127001 (2010).

18. "High speed InGaAs photodiode on ceramic submount," ELUXI Ltd., http://www.eluxi.co .uk/ingaashighspeedonceramic.html.

19. K. Wasa et al., "Patterning vertical sidewall using standard aligner," in 31th Int. Conf. Micro Electro Mech. Syst., Belfast, pp. 475-478 (2018).

20. S. Kumagai, H. Kubo, and M. Sasaki, "Light-absorbent liquid immersion angled exposure for patterning three-dimensional samples with vertical sidewalls," J. Micromech. Microeng. 27, 025016 (2017).

21. S. Kumagai, H. Tajima, and M. Sasaki, "Flow analysis of photoresist spray coating towards improving coverage on three-dimensional structures," Jpn. J. Appl. Phys. 50, 106501 (2011).

22. V. G. Kadajji and G. V. Betageri, "Water soluble polymers for pharmaceutical applications," Polymers 3, 1972-2009 (2011).

23. M. Sasaki, H. Yuge, and H. Suzuki, "Fine patterning method for roll surface using photolithography - micro-fabrication of relief for texturing-," J. Jpn. Soc. Technol. Plast. 60(702), 195-202 (2019). 
Minoru Sasaki received his MS and Dr Eng degrees from Nagoya University in 1993 and 1995, respectively. In 1995, he was a research fellow of the Japan Society for the Promotion of Science. Since 1996, he has been an assistant professor at Tohoku University. Since 2007, he has been a professor at Toyota Technological Institute, Nagoya, Japan. His current research interests include optical MEMS and 3D micro-fabrication.

Biographies of the other authors are not available. 